\title{
Neural Correlates of Motor Learning in the Vestibulo-Ocular Reflex: Dynamic Regulation of Multimodal Integration in the Macaque Vestibular System
}

\author{
Soroush G. Sadeghi, ${ }^{1}$ Lloyd B. Minor, ${ }^{2}$ and Kathleen E. Cullen ${ }^{1}$ \\ 'Department of Physiology, McGill University, Montreal, Quebec H3G 1Y6, Canada, and 2Department of Otolaryngology-Head and Neck Surgery, The \\ Johns Hopkins University School of Medicine, Baltimore, Maryland 21287
}

\begin{abstract}
Motor learning is required for the reacquisition of skills that have been compromised as a result of brain lesion or disease, as well as for the acquisition of new skills. Behaviors with well characterized anatomy and physiology are required to yield significant insight into changes that occur in the brain during motor learning. The vestibulo-ocular reflex (VOR) is well suited to establish connections between neurons, neural circuits, and motor performance during learning. Here, we examined the linkage between neuronal and behavioral VOR responses in alert behaving monkeys (Macaca mulatta) during the impressive recovery that occurs after unilateral vestibular loss. We show, for the first time, that motor learning is characterized by the dynamic reweighting of inputs from different modalities (i.e., vestibular vs extravestibular) at the level of the single neurons that constitute the first central stage of vestibular processing. Specifically, two types of information, which did not influence neuronal responses before the lesion, had an important role during compensation. First, unmasked neck proprioceptive inputs played a critical role in the early stages of this process demonstrated by faster and more substantial recovery of vestibular responses in proprioceptive sensitive neurons. Second, neuronal and VOR responses were significantly enhanced during active relative to passive head motion later in the compensation process ( $>3$ weeks). Together, our findings provide evidence linking the dynamic regulation of multimodal integration at the level of single neurons and behavioral recovery, suggesting a role for homeostatic mechanisms in VOR motor learning.
\end{abstract}

\section{Introduction}

Motor learning is essential not only for the acquisition of new skills but also the reacquisition of formerly mastered skills that have been compromised as a result of brain lesion or disease. Understanding the changes that occur during learning is a fundamental problem in neuroscience, and the relative simplicity of the vestibulo-ocular reflex (VOR) is particularly well suited to establishing links between neurons, neural circuits, and motor performance. The VOR is mediated by a three neuron pathway: the vestibular nerve transmits sensory information to neurons in the vestibular nuclei, which directly project to motoneurons that drive eye motion (Lorente de Nó, 1933). The compensatory eye movements produced by the VOR stabilize images on the retina to prevent blurred vision during the head movements made in everyday activities.

\footnotetext{
Received March 11, 2010; revised June 7, 2010; accepted June 15, 2010.

This work was supported by grants from the Canadian Institutes of Health Research and National Institutes of Health Grant R01 DC02390. We thank Drs. Maurice Chacron, Paul Fuchs, Elisabeth Glowatzki, and Charley Della Santina for critically reading this manuscript. Marion Van Horn, Jessica Brooks, Diana Mitchel, Corentin Massot, Mohsen Jamali, and Jerome Carriot provided useful comments. Diana Mitchel and Marion Van Horn helped in preparing the figures.

Correspondence should be addressed to Kathleen E. Cullen, Department of Physiology, McGill University, 3655 Sir William Osler, Montreal, Quebec H3G 1Y6, Canada. E-mail: kathleen.cullen@mcgill.ca.

S. G. Sadeghi's present address: Department of Otolaryngology-Head and Neck Surgery, The Johns Hopkins University School of Medicine, Baltimore, MD 21287.

DOI:10.1523/JNEUROSCI.1368-10.2010

Copyright $\odot 2010$ the authors $\quad 0270-6474 / 10 / 3010158-11 \$ 15.00 / 0$
}

Changes in environmental requirements, such as those brought about by the magnifying lens worn to correct myopia, lead to impressive VOR adaptation (Shelhamer et al., 1992). Similarly, the VOR shows remarkable plasticity in response to the effects of aging, disease, and trauma to the nervous system (for review, see Cullen, 2008). Long-term potentiation (LTP) and long-term depression (LTD) are widely viewed as playing critical roles in recalibrating the efficacy of vestibular transmission through VOR pathways (Caria et al., 2001; Grassi et al., 2001). However, recent studies have emphasized that learning can be mediated by multiple processes, including homeostatic mechanisms that operate over longer timescales, in addition to rapid Hebbian mechanisms (for review, see Feldman, 2009). Notably, experimentally induced changes in network activity (ranging from hours to days) produce long-term changes in the strength of sensory neocortical synapses (Kotak et al., 2005; Maffei and Turrigiano, 2008). Correspondingly, chronic peripheral vestibular loss can induce changes in synaptic strength onto vestibular nuclei neurons (Goto et al., 2000, 2001) as well as alterations in neuronal membrane properties (Beraneck et al., 2003, 2004).

To date, homeostatic plasticity has been primarily characterized in slice cultures (in vitro) or reduced preparations. Thus, the question of how homeostatic plasticity contributes to motor learning remains open. We hypothesized that activity-dependent synaptic scaling would drive the relative reweighting of inputs from different modalities (i.e., vestibular vs extravestibular) to restore network activity to a set point level after vestibular loss. To 
test this, we recorded from single VOR interneurons in the contralesional vestibular nuclei and determined whether sensitivities to vestibular and/or extravestibular signals changed in parallel with improvements in motor performance. We found that, although vestibular sensitivities were markedly reduced immediately after lesion, extravestibular signals - not present before the lesion-were unmasked. Initially, neck proprioceptive inputs played a key role in the compensation process. At later stages, enhanced neuronal responses during active compared with passive head motion paralleled improvements in motor performance, consistent with the integration of a motor efference copy information at the first central stage of vestibular processing. Thus, our results show that multimodal integration can be dynamically regulated in the vestibular system and strongly favor a causal role for homeostatic plasticity in motor learning.

\section{Materials and Methods}

Subjects and surgery. Experiments were performed on two male rhesus macaque monkeys (Macaca mulata) weighing $\sim 8 \mathrm{~kg}$. The animals were chronically implanted with a post for head restraint, recording chamber, and scleral search coils for high-resolution eye movement recording as described previously (Sadeghi et al., 2007b). After the surgery, the animals were administered buprenorphine $(0.01 \mathrm{mg} / \mathrm{kg}$, i.m. ) for postoperative analgesia, and the antibiotic Cephazolin (Ancef; $25 \mathrm{mg} / \mathrm{kg}$, i.m., for $5 \mathrm{~d}$ ). Animals were trained using standard operant conditioning to fixate visual targets for a juice reward. In both animals, we recorded from single units directly after training as well as after unilateral labyrinthectomy. Labyrinthectomy was performed through the mastoid bone to remove the ampulla of the three semicircular canals, the utricle and saccule, and the distal ends of the ampullary nerve branches (Sadeghi et al., 2006). All procedures were approved by the McGill University Animal Care Committee and The Johns Hopkins University Animal Care and Use Committee and were in compliance with the guidelines of the Canadian Council on Animal Care and the National Institutes of Health.

Experimental design and data acquisition. Monkeys were initially head restrained during experiments and yaw rotations about the earth vertical axis were applied using a motion stimulator, located within a $1 \mathrm{~m}^{3}$ magnetic field coil (CNC Engineering). A visual target (HeNe laser) was projected, via a system of two galvanometer-controlled mirrors, onto a cylindrical screen located $60 \mathrm{~cm}$ away from the monkey's head. Monkeys were trained to follow the visual target, and neuronal sensitivities to saccades, ocular fixation, and pursuit were characterized by having the monkey follow target motion that (1) stepped between horizontal positions over a range of $\pm 30^{\circ}$ and (2) moved sinusoidally $(0.5 \mathrm{~Hz}, \pm 40 \% \mathrm{~s}$ peak velocity). Target and turntable motion were controlled by a UNIXbased real-time data acquisition system (REX) (Hayes et al., 1982).

The experimental design consisted of four stimulus conditions. First, to stimulate the vestibular system, monkeys were rotated about an earth vertical axis with their heads restrained $\left(0.5 \mathrm{~Hz}\right.$, peak velocity of $\left.\pm 40^{\circ} / \mathrm{s}\right)$ both in the dark (whole-body rotation) and while they suppressed their VOR by fixating a visual target that moved with the vestibular turntable (i.e., VOR cancellation condition). Second, to stimulate neck proprioceptors, the monkey's head was held stationary relative to the earth while its body was sinusoidally $(0.5 \mathrm{~Hz}, \pm 40$ or $\pm 80 \%$ s $)$ rotated beneath. Third, combined stimulation of the vestibular system and neck proprioceptors was induced by passively rotating the monkey's head on its body using a torque motor (Kollmorgen) attached to the head (Huterer and Cullen, 2002; Sadeghi et al., 2006, 2007a,b, 2009). The applied stimulation produced horizontal sinusoidal head rotations about the vertical axis, relative to a stationary body $(1 \mathrm{~Hz}, \pm 40 \%$ s), as well as passive rotations of the head relative to the body that had trajectories comparable with those produced during actively generated orienting gaze shifts. Finally, the monkey's head was slowly and carefully released so that it was free to make active head movements (i.e., horizontal rotations about the earth vertical axis) so that the responses of the same neuron could then be recorded during voluntary (i.e., active) horizontal gaze shifts toward targets, as described previously (Roy and Cullen, 2002).
Electrophysiology. Extracellular single-unit recordings were performed using enamel-insulated tungsten microelectrodes (7-10 $\mathrm{M} \Omega$ impedance; Frederick Haer) advanced into the brainstem through a transdural guide tube using a lightweight microdrive (Narishige). Single neurons were isolated using a conventional amplifier system and bandpass eight pole filter $(400 \mathrm{~Hz}$ to $10 \mathrm{kHz})$. The abducens nucleus was first identified based on its stereotypical discharge patterns during eye movements (Cullen et al., 1993; Sylvestre and Cullen, 1999) and then used as a landmark to determine the location of the medial and lateral vestibular nuclei. Position-vestibular-pause (PVP) neurons were then identified on the basis of their characteristic physiological response properties including oppositely directed sensitivities to vestibular stimulation and eye position, and cessation of firing (pause) during rapid saccadic eye movements (Roy and Cullen, 1998). We made our recordings in the contralesional vestibular nuclei, since the results of previous in vitro studies had suggested greater compensation compared with lesioned side (for review, see Straka et al., 2005). In addition, we specifically focused on neurons that mainly receive inputs from the horizontal canals and divided them into two groups (Duensing and Schaefer, 1958): type I neurons (that receive excitatory inputs from the ipsilateral horizontal canal) and type II neurons. Notably, this latter class of neurons is known to receive excitatory input from contralateral type I neurons and in turn provides the major inhibitory input to ipsilateral type I neurons (Shimazu and Precht, 1966; Malinvaud et al., 2010).

Gaze and head position were monitored using the magnetic search coil technique, and turntable velocity was measured using an angular velocity sensor (Watson). Single-unit responses, horizontal and vertical gaze and head positions, target position, and table velocity were recorded on a digital audio tape for later playback. Action potentials were discriminated during playback using a windowing circuit (BAK) that was manually set to generate a pulse coincident with the rising phase of each action potential. In addition, gaze position, head position, target position, and table velocity signals were low-pass filtered at $250 \mathrm{~Hz}$ (eight pole Bessel filter) and sampled at $1 \mathrm{kHz}$.

Data analysis. Data were imported into the Matlab (The MathWorks) programming environment for analysis. Recorded gaze and head position signals were digitally filtered with zero-phase at $125 \mathrm{~Hz}$ using a 51st order FIR (finite-impulse-response) filter with a Hamming window. Eye position was calculated from the difference between gaze and head position signals. Gaze, eye, and head position signals were digitally differentiated to produce velocity signals. Neuronal responses were represented using a spike density function in which a Gaussian was convolved with the spike train (SD, $10 \mathrm{~ms}$ for sinusoidal rotations; SD, $5 \mathrm{~ms}$ for gaze shifts) (Cullen et al., 1996; Sylvestre and Cullen, 2006). Statistical significance was determined using Student's $t$ tests.

Control data were obtained by recording eye, head, and table rotations, as well as neuronal responses in the vestibular nuclei of each animal before labyrinthectomy. A labyrinthectomy was then performed on the contralateral side and postlesion data were collected during experimental sessions in which recordings were made in the contralesional vestibular nuclei, starting from day 1 (i.e., 15-28 h) after lesion. Later recordings were made on a weekly basis up to 2 months after lesion.

To quantify behavioral performance, we calculated the gain of the VOR response during sinusoidal rotations for full cycles as well as for ipsilateral and contralateral half-cycles of rotation. For the latter analysis, the head velocity signal was divided into right and left half-cycles based on zero crossings of the stimulus. At least 10 cycles of rotations were analyzed for each measurement. VOR and cervico-ocular reflex (COR) gains were calculated as the resultant slow phase (i.e., desaccaded) eye velocity divided by the turntable velocity after accounting for the phase difference (Sadeghi et al., 2006). In addition, to compute the gain of VOR during active head movements, eye responses were characterized during head motion made both before the period during which gaze was redirected, as well as during the $10-80 \mathrm{~ms}$ period after the end of the gaze shift (i.e., postgaze shift period), in which the gaze was stable but the head continued to move.

The recordings of neural responses concentrated on the functionally distinct group of cells in the vestibular nuclei classified in previous experiments (Roy and Cullen, 1998) as PVP neurons. To identify PVP cells, 
neuronal eye position sensitivities [ $k$, (spikes/second)/degree] were computed from periods of fixation using a multiple-regression analysis (Roy and Cullen, 1998). Spike trains were also assessed to verify that neurons paused during saccades. In addition, a least-squared regression analysis was used to determine the response of each unit to vestibular stimulation during passive whole-body rotations as follows:

$$
\hat{\mathrm{fr}}(t)=b+\mathrm{Sv}_{\text {vest }} \dot{H}(t)+\mathrm{Sa}_{\text {vest }} \ddot{H}(t)+k E(t),
$$

where $\hat{f r}$ is the estimated firing rate, $\mathrm{Sv}_{\text {vest }}$ and $\mathrm{Sa}_{\mathrm{vest}}$ are coefficients representing sensitivities to head velocity and acceleration, $b$ is a bias term, $E$ is eye position, and $\dot{H}$ and $\ddot{H}$ are head velocity and head acceleration, respectively. Only unit data from periods of slow-phase eye velocity that occurred between quick phases of vestibular nystagmus and/or saccades were included in the analysis. The estimated coefficients $S_{v}$ vest and $\mathrm{Sa}_{\text {vest }}$ were then used to calculate the modulation sensitivity [(spikes/ second)/(degrees/second)] and phase shift (degrees) relative to head velocity of each unit (Sadeghi et al., 2009).

A comparable approach was next used to describe the response of each unit to neck proprioceptive stimulation during passive rotation of the body under a stationary head. To quantify neuronal responses, we determined the best estimate of the sensitivity of each neuron to neck rotation using the following equation:

$$
\hat{\mathrm{fr}}(t)=b+\mathrm{Sv}_{\text {neck }} \dot{B}(t)+\mathrm{Sa}_{\text {neck }} \ddot{B}(t)+k E(t),
$$

where $S v_{\text {neck }}$ and $\mathrm{Sa}_{\text {neck }}$ are coefficients representing sensitivities to neck (=body or equivalently the vestibular turntable) velocity and acceleration, and $\dot{B}$ and $\ddot{B}$ are body velocity and acceleration, respectively. Because neuronal responses typically led rather than lagged body velocity, our formalization of the model included velocity and acceleration terms. Similar to vestibular sensitivities, the estimated coefficients were then used to calculate the modulation sensitivity of each unit [(spikes/second)/(degrees/second)] and phase shift (degrees) relative to velocity of body rotation (Sadeghi et al., 2009).

Finally, we used a similar approach for the characterization of responses during combined vestibular and proprioceptive stimulation evoked by passive sinusoidal head-on-body rotations (i.e., the combined condition). Neuronal responses were estimated using the following equation:

$$
\hat{\mathrm{fr}}(t)=b+\mathrm{Sv}_{\mathrm{hob}} \dot{H} B(t)+\mathrm{Sa}_{\mathrm{hob}} \ddot{H} B(t)+k E(t),
$$

where $S v_{h o b}$ and $S a_{\text {hob }}$ are coefficients representing sensitivities to headon-body velocity and acceleration, $\dot{H B}$ and $\ddot{H B}$ are head-on-body velocity and acceleration, respectively. Estimated sensitivities $\left(\mathrm{Sv}_{\mathrm{hob}}\right.$ and $\mathrm{Sa}_{\mathrm{hob}}$ ) were then compared with those predicted based on the linear summation of the vestibular and proprioceptive sensitivities estimated for the same neuron during whole-body rotations (Eq. 1) and bodyunder-head rotations (Eq. 2), respectively.

To quantify the ability of the linear regression analyses to model neuronal discharges during each paradigm, we computed the variance-accounted-for (VAF) provided by each regression equation (Cullen et al., 1996). The VAF was defined as follows: $\{\mathrm{VAF}=1-[\operatorname{var}(\hat{\mathrm{fr}}-\mathrm{fr}) / \operatorname{var}(\mathrm{fr})]\}$, where $\hat{\mathrm{fr}}$ represents the modeled firing rate (i.e., regression equation estimate) and $\mathrm{fr}$ represents the actual firing rate.

\section{Results}

To investigate the contributions of different modalities (i.e., vestibular vs extravestibular) to reestablishing network function after vestibular loss, we not only need to separately assess the contribution of each signal to neuronal responses but also determine whether the observed changes lead to improvements in motor performance (i.e., VOR compensation). We begin by considering the linkage between changes in neuronal vestibular sensitivities and simultaneously measured VOR responses. We then determine whether sensitivities to extravestibular signals are unmasked in parallel with modality-specific improvements in motor performance. Finally, we address whether dynamic regulation

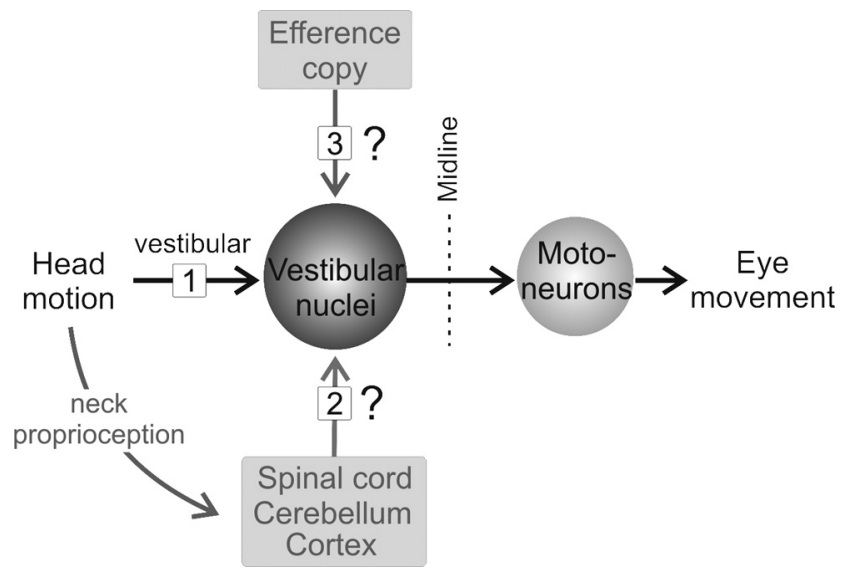

Figure 1. Schematic diagram of the main direct pathway that mediates the VOR. Primary sensory afferents (input 1) send vestibular signals to the PVP neurons in the vestibular nuclei. These neurons in turn project to the extraocular motoneurons on the contralateral side to produce VOR eye movements. In addition, self motion information arising from the activation of proprioceptors (input 2) and/or motor efference copy information (input 3) could help drive compensation at this site.

of multimodal integration is associated with increases in the recovery of vestibular sensitivity by individual neurons during this motor learning.

\section{Neural correlates of compensation: vestibular responses}

We directly measured the efficacy of VOR pathways during vestibular stimulation by simultaneously recording motor performance and the single-unit responses of the individual neurons in the vestibular nuclei (Fig. 1, input 1), which constitute the intermediate leg of the direct VOR pathway (McCrea et al., 1987; Scudder and Fuchs, 1992; Cullen and McCrea, 1993). These VOR neurons receive a strong monosynaptic drive from the ipsilateral nerve and, in turn, project directly to contralateral extraocular motoneurons. They can be easily identified by their characteristic responses (i.e., an increase in their activity as a function of contralateral eye position and ipsilateral head velocities) and are called type I PVP neurons. Recordings were also made from type II PVP neurons, which contribute to the commissural pathways that interconnect the vestibular nuclei on each side (Shimazu and Precht, 1966; Malinvaud et al., 2010). These neurons are characterized by oppositely directed head and eye movement sensitivities to those of type I PVP neurons (Roy and Cullen, 2002).

To assess sensitivities to vestibular inputs, we first quantified the behavioral performance recorded during passive whole-body rotations $\left(0.5 \mathrm{~Hz}, 40^{\circ}\right.$ s $)$ before and after unilateral labyrinthectomy. Before unilateral labyrinthectomy, eye movement responses were fully compensatory (Fig. 2A1, top row). However, immediately after lesion, VOR responses were reduced and asymmetric, characterized by diminished responses to rotations toward the side of the lesion (Fig. 2A1, middle row). Finally, when measured 4 weeks after lesion, VOR responses evoked by rotations toward the side of the lesion remained defective, whereas responses evoked by rotations in the opposite direction appeared normal (Fig. $2 A 1$, bottom row).

To quantify behavioral performance, we computed the average gain of the VOR eye movement response (see Materials and Methods) for both animals before and after lesion (Fig. 2 A2). On the day after lesion, gains of responses evoked by rotations in either direction were dramatically reduced to $70-80 \%$ relative to control values. However, responses to both ipsilesionally and 
A1
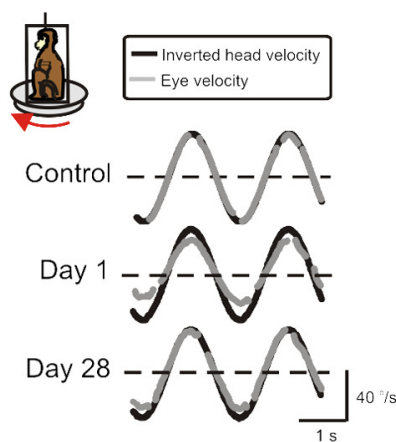

$\mathrm{A} 2$

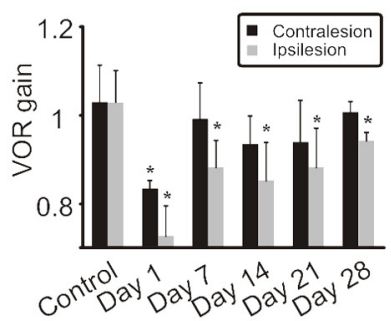

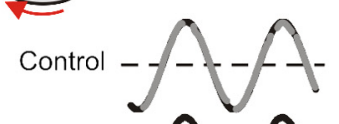

B1

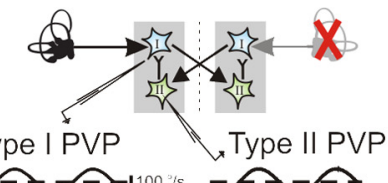
Head velocity

Control

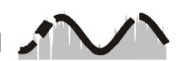

Day 1
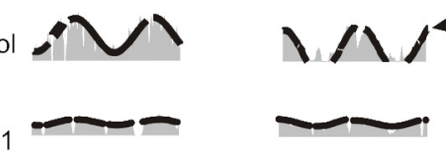

Day 28
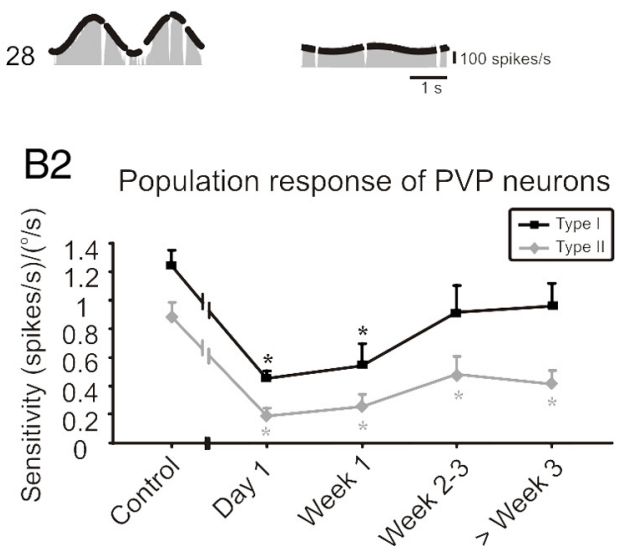

Figure 2. Changes in simultaneously measured VOR and neuronal responses after unilateral labyrinthectomy. $\mathbf{A 1}$, Example VOR responses show reduced and asymmetric gains immediately after lesion (day 1), and impressive compensation, recovering to nearly normal values by day 28 . Note that head velocity traces have been inverted to facilitate comparison with the evoked eye velocities. A2, VOR gains averaged across both animals. On day 1, responses were reduced for both ipsilaterally and contralaterally directed rotations. Over the next 2-3 weeks, contralesional gains improved to normal values and ipsilesional gains were nearly compensatory. B1, Examples of type I and type II PVP responses before and at different time points after contralateral labyrinthectomy. Response of both cell types decreased significantly immediately after lesion (day 1). Whereas the sensitivity of type I neurons improved over time reaching normal values by day 28 , that of type ll neurons did not show significant improvement $(p>0.1)$. The inset shows that type I neurons receive indirect inputs from contralateral labyrinth through inhibitory type II neurons. B2, Summary of the analysis of (1) the population of 57 neurons (40 type I and 17 type II) recorded under control conditions and (2) the population of 109 neurons recorded after lesion ( 56 type l and 53 type II). 0 f the latter group, 44 were recorded on the first day (i.e., $15-28 \mathrm{~h}$ ) after lesion, 32 in the period of $7-21 \mathrm{~d}$ after lesion, and 33 in the 1-2 months after lesion. The asterisk ${ }^{*}$ ) represents significant difference with regard to control (i.e., before lesion), $p<0.05$. Error bars indicate SEM.

contralesionally directed rotations improved over the next month, with contralesional responses fully recovering to normal levels by day $7(p<0.01)$.

Having established that the VOR shows robust compensation after vestibular unilateral labyrinthectomy, we next quantified the corresponding responses of vestibular nuclei neurons. Based on the circuitry of the VOR pathways (Fig. 2B1), we predicted that type II as well as type I PVPs in the contralesional vestibular nuclei should show a decreased sensitivity to vestibular stimulation. Both neurons lose inputs that they normally receive from the lesioned nerve via the commissural connections between the two vestibular nuclei; for type II neurons, this input is direct, whereas for type I neurons it is mediated mostly via type II neurons (Shimazu and Precht, 1966; Malinvaud et al., 2010).

Figure $2 B 1$ (control) shows the robust responses of an example type I PVP neuron before lesion. Consistent with our prediction, the sensitivity of neurons decreased dramatically immediately after contralateral labyrinthectomy (Fig. 2B1, day $1)$. Strikingly, however, this diminished response nearly recovered to normal values over the following weeks (Fig. 2 B1, day 28). Sensitivity of type II neurons similarly decreased immediately after lesion. However, in contrast to type I neurons, the responses of type II neurons never fully recovered. This is illustrated for the example neuron shown in Figure 2B1 (right column). Even 4
Estimate

weeks after lesion, this type of neuron was typical in that its response to vestibular stimulation was far less robust than observed before the lesion.

Figure $2 \mathrm{~B} 2$ shows the time course of the change in vestibular sensitivity of the population of neurons before $(n=57)$ and on different days after lesion $(n=$ 109). The average sensitivities of neurons recorded in control animals were $1.2 \pm$ 0.1 and $0.9 \pm 0.1$ (spikes $/ \mathrm{s}) /(\%)$, for type I and II neurons, respectively, similar to those reported in previous studies (Roy and Cullen, 1998, 2002). Immediately after contralateral labyrinthectomy (day 1), the average sensitivity of both type I and type II neurons dramatically decreased $(>50 \%$ reduction; $p<0.0001)$ reaching values of $0.45 \pm 0.05$ and $0.19 \pm 0.06$ (spikes/s)/(\%), respectively. In the following weeks, the responses of type I neurons improved, so that their average vestibular sensitivity reached normal values by weeks $2-3$ after lesion [0.92 \pm 0.19 (spikes/s) $/(\%$ s); $p=0.12]$. Although type II neurons showed a slight improvement in their responses, their sensitivities never reached normal values, even $60 \mathrm{~d}$ after lesion $[>50 \%$ reduction in sensitivity; $0.41 \pm 0.1$ (spikes $/ \mathrm{s}) /(\% / \mathrm{s}) ; p=0.0002]$. Together, these results from lesioned animals indicate that increased weighting of the excitatory input to type I neurons (i.e., via direct input from the intact contralesional vestibular nerve) provides a robust substrate to mediate compensation. In contrast, type II neurons show little recovery consistent with the fact that the source of the excitatory inputs to these cells is the contralateral nerve, which had been lesioned.

\section{Neural correlates of compensation: the unmasking of extravestibular inputs}

The results shown so far demonstrate a strong relationship between changes in the vestibular sensitivity of single neurons and the recovery of motor performance after lesion. However, these findings consider the VOR as a unimodal pathway, since changes in neuronal sensitivities and behavioral performance were only characterized for vestibular stimulation. Because the vestibular system, unlike other senses, is multisensory and multimodal immediately at the first central stage of processing, we next tested whether information about self motion derived from sources other than the vestibular sensors also plays an important role in compensation. Notably, when head movements are made in a natural context, the brain has access to proprioceptive and motor-related signals as well as vestibular information. If neuronal sensitivities to stimulation of proprioceptive inputs (Fig. 1, input 2) and/or the production of motor commands resulting in self motion (Fig. 1, input 3) showed changes that parallel improvements in motor performance during compensation, then we could conclude that the reweighting of extravestibular inputs at the first stage of central vestibular processing induces motor recovery. 
A

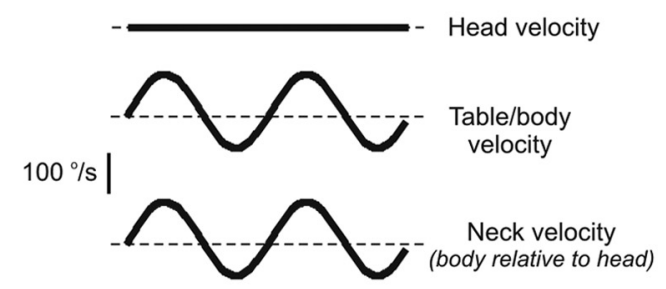

B

E

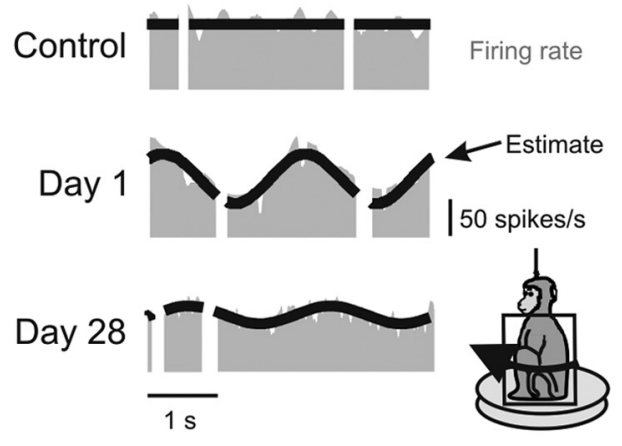

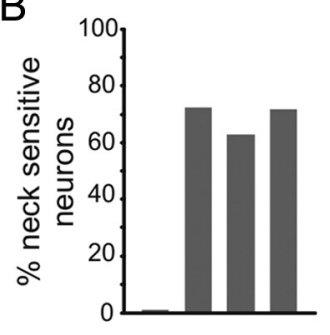

C

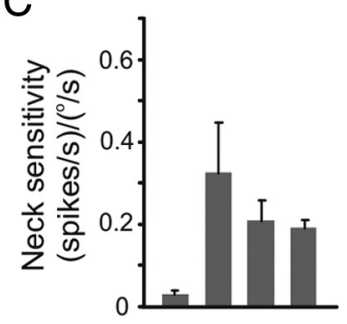

F

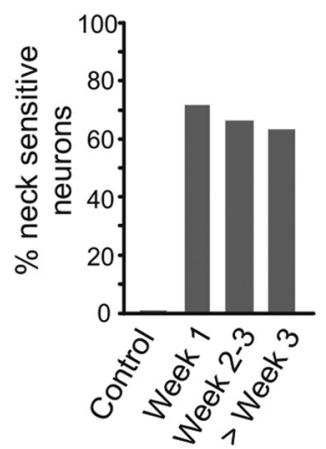

D

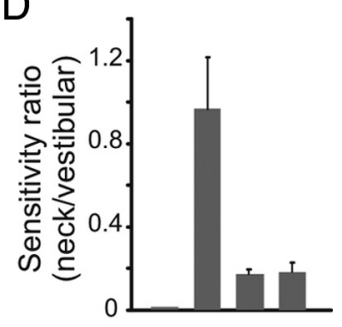

G

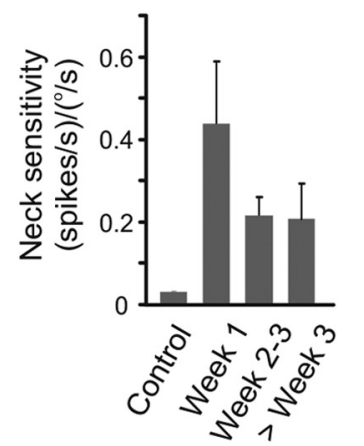

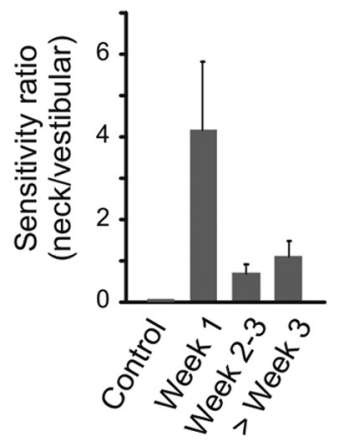

Figure 3. The majority of PVP neurons show robust modulation to stimulation of neck proprioceptors after contralateral labyrinthectomy. $A$, Examples of type I neuronal responses during stimulation of neck proprioceptors. In intact animals, neurons are not sensitive to stimulation. In contrast, the example neuron shown on day 1 after the lesion responded robustly to neck stimulation. The neuron shown on day 28 also responded to neck stimulation, but with a lower sensitivity. $\boldsymbol{B}$, The percentage of neck-sensitive type I neurons remained constant (60-70\%) from week 1 to 8 after lesion. C, The average of the absolute values of neck sensitivities of type I neurons decreased during compensation, but never reached control values (i.e., responses remained significant). $\mathbf{D}$, Decreases in neck sensitivity of type I neurons were temporally linked to increases in the vestibular sensitivities. Accordingly, neck sensitivities were the most robust the first week after lesion. $\boldsymbol{E}$, The percentage of neck-sensitive type II neurons remained constant (60-70\%) from week 1 to 8 after lesion $(n=29)$. $\boldsymbol{F}$, The average of the absolute values of neck sensitivity in type II neurons decreased from $0.44 \pm 0.15$ on week 1 after lesion to $0.22 \pm 0.05$ and $0.20 \pm 0.08$ during weeks $2-3$ and after week 3 , respectively. $\mathbf{G}$, The ratio of neck and vestibular sensitivities shows that neck sensitivities of type II neurons were the most robust the first week after lesion. Error bars indicate SEM.

First, to test whether proprioceptive information could potentially be used to support compensation, we recorded from single neurons before and after lesion during a paradigm in which proprioceptive stimulation was delivered in isolation (see Materials and Methods). Figure $3 A$ illustrates the responses recorded from three typical type I PVP neurons while we sinusoidally rotated the monkey's body beneath its earth-stationary head. As previously shown by Roy and Cullen (2001), type I PVP neurons did not respond to the passive stimulation of neck proprioceptors before labyrinthectomy (Fig. 3A, control). Strikingly, however, immediately after lesion, the majority of neurons $(>70 \%)$ showed robust modulation in response to the identical stimulation (Fig. 3A, day 1). When tested a month after lesion, the majority of neurons remained sensitive to the stimulation of neck proprioceptors, but responses were far less striking than those measured on day 1 (Fig. 3A, day 28). Figure $3 B$ shows the percentage of neck-sensitive neurons across the population as a function of time. Although this percentage showed little change after lesion, neck sensitivities, which were nonexistent in control animals, peaked just after lesion and decreased significantly by the second week after lesion (Fig. $3 C)(p<0.05$; note, population values were computed from the average of absolute values since the polarity of the responses differed across individual neurons).

Additional analysis revealed that the observed decreases in neck sensitivities were associated with a coincident increase in the vestibular sensitivity of type I PVP neurons. To quantify this observation, we computed the ratio between neck and vestibular sensitivities for all neurons recorded after lesion that were sensitive to neck proprioceptor stimulation. The trends across time (Fig. 3D) show that neck inputs made the greatest contribution immediately after the lesion. Overall, similar findings were also obtained in the analysis of the percentages and sensitivities of neck-sensitive type II neurons after lesion (Fig. $3 E-G$ ).

\section{Relationships between neural sensitivities to proprioception and vestibular compensation}

The presence of neck proprioceptive responses on VOR interneurons was observed immediately after but never before labyrinthectomy. The fact that VOR interneurons show significant modulation in response to stimulation of proprioceptors after lesion, suggests that the unmasking of this extravestibular input plays a role in the compensation process. To further investigate this possibility, we examined two possible, non-mutually exclusive roles, namely that the unmasking of neck proprioceptive inputs (1) results in the enhancement of neck-driven ocular responses to improve gaze stabilization and/or (2) reflects a homeostatic mechanism that ensures continued dynamic stimulation of individual neurons after lesion.

Proprioceptive driven ocular responses, such as the COR, do not make significant contribution to gaze stabilization in normal subjects (Dichgans et al., 1973; Bronstein and Hood, 1986; Jürgens and Mergner, 1989; Roy and Cullen, 2002). If the unmasking of proprioceptive inputs enhanced neck-driven ocular responses to compensate for the defective VOR, then we would have expected more robust behavioral responses during neck proprioceptive stimulation after vestibular lesion. This prediction was contradicted by our quantification of behavioral performance (i.e., the cervico-ocular reflex) measured during the same paradigms used to compute neuronal neck sensitivity above (i.e., as in Fig. 3). Average behavioral gains were computed by measuring eye movements evoked by sinusoidal rotation of the monkey's body beneath its earth-stationary head. Figure $4 A 1$ shows 

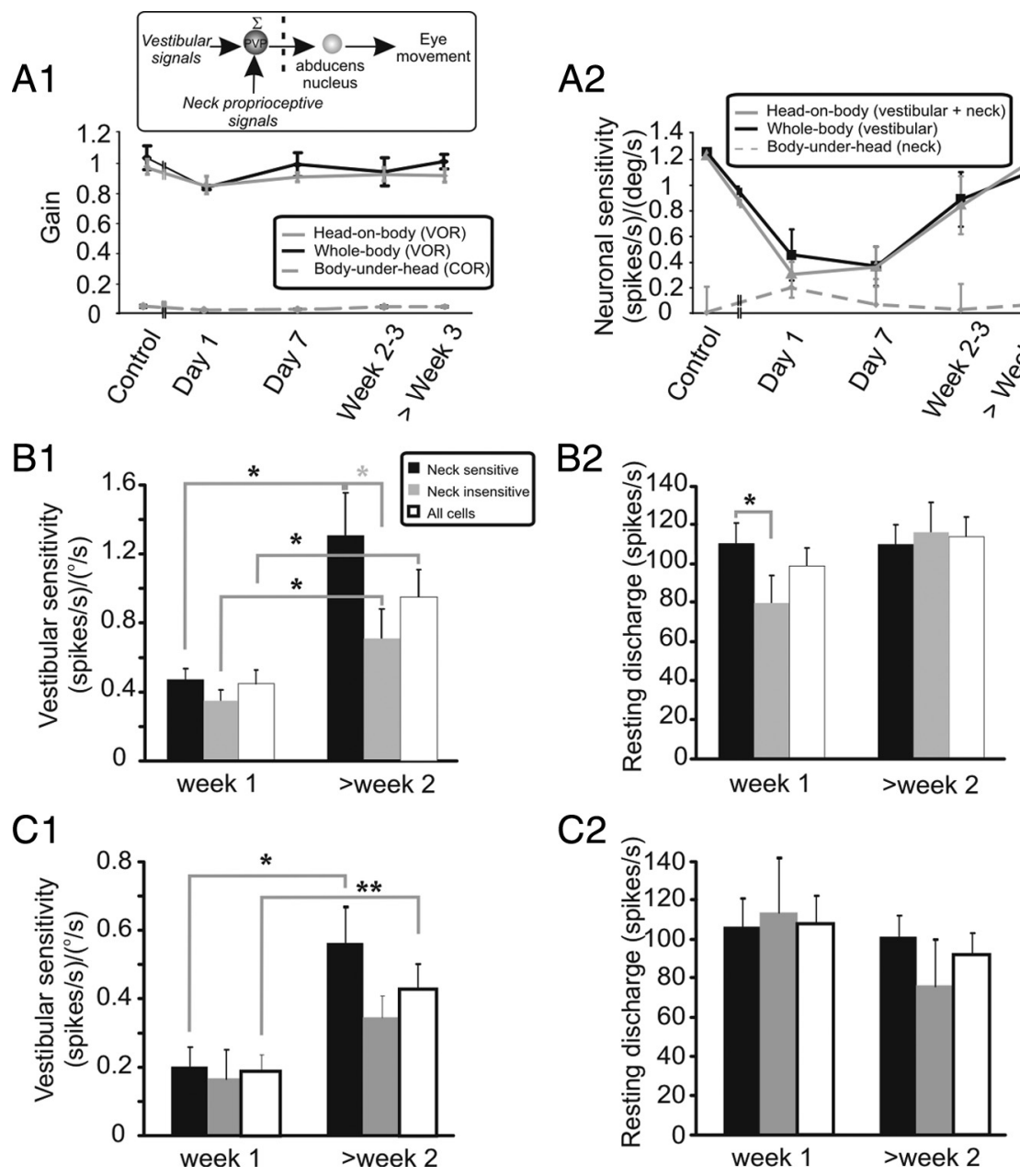

Figure 4. The role of neck inputs in vestibular compensation of PVP neurons. A1, The presence of neck proprioceptive responses on type I neurons did not enhance the COR (dashed gray line); response gains were negligible before and after lesion. Similarly, the VOR responses evoked by combined vestibular and neck stimulation (head-on-body rotation, gray line) and vestibular stimulation alone (whole-body, black line) were comparable. A2, Average neuronal sensitivities computed for the population of type I neurons during the same paradigms as in $\mathbf{A 1}$. Note that the direction of neck sensitivities was accounted for in the calculation (i.e., sensitivities to rightward vs leftward stimulation were considered as positive and negative values, respectively). As can be seen by the average sensitivities to body-under-head rotation (dashed line) and the lack of difference between head-on-body (black line) and whole-body rotations (gray line), average responses were minimal even immediately after lesion. $\mathbf{B 1}$, The vestibular sensitivities of neck-sensitive type I neurons showed more substantial (gray asterisk) recovery over time than neck-insensitive neurons. B2, The resting discharge of neck-sensitive type I neurons initially showed better recovery over time, compared with neckinsensitive neurons. However, later in the compensation process (more than week 2), both groups showed comparable resting discharges. C1, The vestibular sensitivities of neck-sensitive type II neurons $(n=29)$ showed more substantial recovery over time, than neck-insensitive neurons $(n=21)$. As a result, the entire population of neurons also showed a significant increase in their sensitivities $>2$ weeks after lesion. $\mathbf{C}$, The resting discharges of neck sensitive and insensitive neurons were similar at all times after lesion and did not differ from control values ( $t$ test, $p>0.2)$. The single asterisk $\left(^{*}\right)$ and double asterisk $\left(^{* *}\right)$ show significant differences ( $t$ test) at $p<0.05$ and $p<0.01$, respectively. Error bars indicate SEM.

the average behavioral performance measured in the two monkeys, before and at different times after unilateral labyrinthectomy (dashed gray line). Neck proprioceptive driven eye movements were negligible before and remained negligible after the lesion.

Given that neck proprioceptive driven ocular reflexes were not enhanced after labyrinthectomy, it follows that eye movements evoked by combined stimulation of the vestibular system and neck proprioceptors should be comparable with those evoked by vestibular stimulation alone. To test this proposal, we also recorded eye movements during head movements made by rotating the head relative to a stationary body. In this condition, PVP neurons in the vestibular nuclei would receive information from neck proprioceptive inputs in addition to their primary input from the vestibular nerve (Fig. $4 A 1$, inset). As expected, the eye movement responses evoked by combined vestibular/proprioceptive stimulation and vestibular stimulation alone (i.e., passive whole-body rotations) were comparable (Fig. 4A1, compare gray and black lines, respectively). Figure $4 A 2$ compares the simultaneously recorded neuronal sensitivities to head-in-space motion for both conditions. Consistent with our behavioral findings, neuronal sensitivities were comparable $(p>0.1)$.

The absence of a cervico-ocular reflex after lesion might appear surprising, considering the high neck proprioceptive sensitivity of PVP neurons in the first week after lesion, particularly of type I neurons that project to the eye motoneurons (Fig. $3 B, C)$. However, when computing the net neck-related command produced by these neurons, it is essential to account for the directional sensitivity of the neck-driven response. In our population, neck-related and vestibular responses were both agonistic and antagonistic. Accordingly, when response direction as well as magnitude was considered, our population of type I PVP neurons showed minimal neck sensitivity acutely after lesion and during the compensation process $[0.19 \pm 0.06$ and $0.04 \pm 0.04$ (spikes/s)/(\%) in week 1 and later, respectively]. Similar qualitative findings were found for type II PVP neurons. Thus, the unmasking of neck-related inputs on VOR interneurons did not result in a parallel change in proprioceptive driven ocular reflex, even though these neurons project directly to the extraocular motor nuclei.

A second possible role for the unmasking of neck proprioceptive inputs is that it supports a homeostatic mechanism that ensures continued dynamic stimulation of individual neurons after lesion. If this were the case, then we would expect that neck-sensitive neurons should show better and/or faster compensation after lesion. Figure 4, B1 and B2, verifies this prediction. The recovery of vestibular sensitivities is compared for neck-sensitive and neck-insensitive neurons during compensation (Fig. 4B1, black and gray bars, respectively; vs all neurons, white bars). Whereas the vestibular sensitivities of both groups of neurons improved significantly over time (black stars; $p<0.04$ ), those of the neck-sensitive neurons showed significantly greater improvement (gray star; $p<0.04)$. Similarly, the resting discharges of neck-sensitive neurons recovered faster compared with those of neck-insensitive neurons, reaching control values (105 \pm 11 spikes/s) during the first week after lesion (Fig. 4B2) $(p<0.05)$. The situation was similar for type II PVPs in that neck-sensitive neurons $(n=29)$ showed significantly better improvement in their vestibular sensitivities compared with neck-insensitive neurons $(n=21)$ (Fig. 
4C1). However, the resting discharge was not different between the two groups of neurons (Fig. 4C2) and control value $(89 \pm 18$ spikes/s).

\section{Unmasking of extravestibular inputs: motor efference copy}

In natural conditions, head movements are commonly self-generated. During these active movements, the brain has access to information about self motion as a result of both the motor command it produces and the resultant stimulation of vestibular and proprioceptive inputs (Fig. 5, compare schematics in the top row). Having established above the relationship between VOR compensation and the unmasking of proprioceptive inputs and changes in vestibular sensitivity of single VOR interneurons, we next asked whether an efferent copy of the head motor command could also contribute to compensation during active head movements (Fig. 1, input 3). Specifically, we quantified the linkage between changes in neuronal response sensitivities and simultaneously measured VOR responses during passive and active head-on-body rotations with comparable trajectories.

Figure 5 shows the responses of two typical type I PVP neurons, one recorded before (Fig. $5 A$ ) and the other recorded 4 weeks after lesion (Fig. 5B). As was the case during passive sinusoidal stimulation, there was excellent correspondence between the optimal fit to the response of the neuron (black line) during passive motion and the prediction computed from the sum of the individual vestibular and proprioceptive response sensitivities of the neuron. This was the case both before and after lesion (left panels; dashed red lines). Similarly, before lesion, the same linear summation prediction well estimated neuronal responses during active head movements (Fig. $5 A$, right panels, dashed red lines). In contrast, after lesion, neuronal responses were underestimated by the linear summation of the sensitivities to vestibular and proprioceptive stimulation of the neuron (Fig. $5 B$, right panels, dashed red lines). Thus, after labyrinthectomy, neurons showed more robust modulation in response to active head than passive head movements. Note that fits were restricted to the neuronal firing associated with active head motion produced before and after the shift in gaze in both conditions, since PVP neurons show a marked pause in firing during the gaze shift portion of active head movements (Roy and Cullen, 1998, 2002).

Figure $6 A$, top panel, summarizes the sensitivities of the population of type I PVP neurons $(n=34)$ recorded before and after lesion during passive (gray) and active (black) movements. Neuronal sensitivities to active and passive head motion were not significantly different before the lesion $(p>0.9)$. Similarly, head motion sensitivities remained comparable in both conditions during the first 3 weeks after lesion. Note, however, that neuronal

A
Passive motion
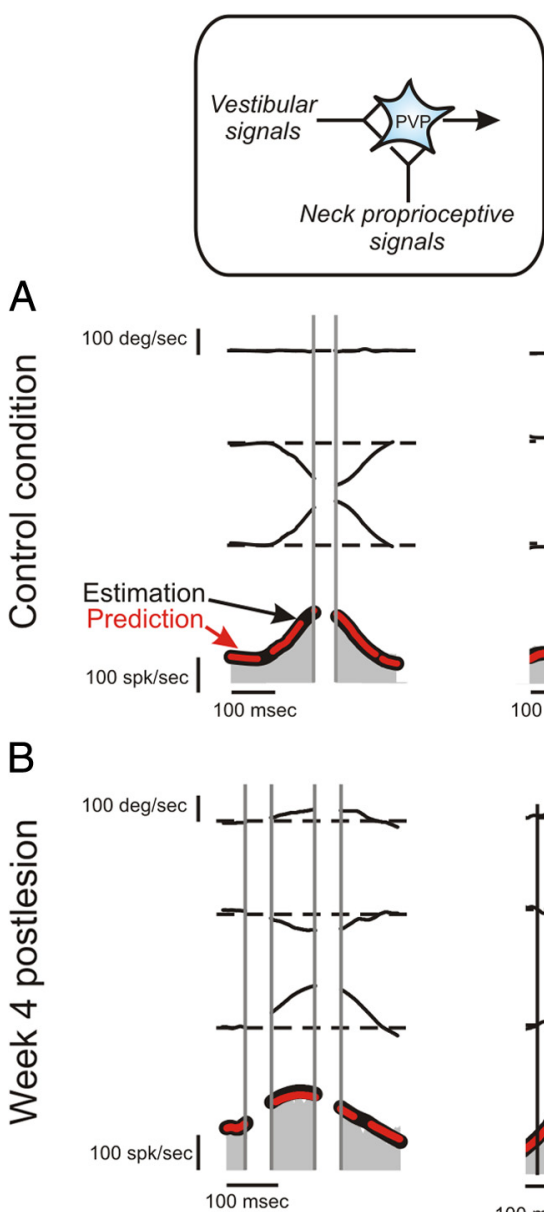

Figure 5. The response of an example type I PVP neurons during passive versus active head-on-body rotations before and ing active motion, and as a result, additional self motion information is potentially available in this condition. For example, this ontrol animals, there was excellent correspondence between the optimal response of the neuron (black line) and the prediction e sum of the vestibular and neck sensitivities of the neuron (red) during both passive and active motion. $\boldsymbol{B}$, In contrast, rediction based on the sum of the vestibular and neck sensitivities of the neuron (red) during passive motion. Notably, neuronal responses were enhanced during active head movements on week 4 after lesion.

head motion sensitivities were significantly elevated 4 weeks after lesion. The mean neuronal sensitivity for our population of type I PVP neurons was $\sim 20 \%$ higher during active compared with passive movements (paired $t$ test, $p<0.05$ ). This difference was consistent across all neurons tested, regardless of the presence or absence of neck sensitivity. In contrast, type II neurons $(n=20)$ did not show a significant change in their sensitivities during active head rotations compared with passive rotations at any time after lesion (Fig. $6 \mathrm{~A}$, bottom panel) (paired $t$ test, $p>0.2$ at each time point). Thus, type I, but not type II, PVP neurons generally fire more robustly in response to active versus passive head movements during the later (i.e., $>3$ weeks) stages of vestibular compensation.

Finally, we asked whether the preferential enhancement of VOR interneuron responses (i.e., type I PVP neurons) contributes to improved behavioral performance during active movements. To address this question, we quantified performance by computing the average gain of the VOR eye movement response 

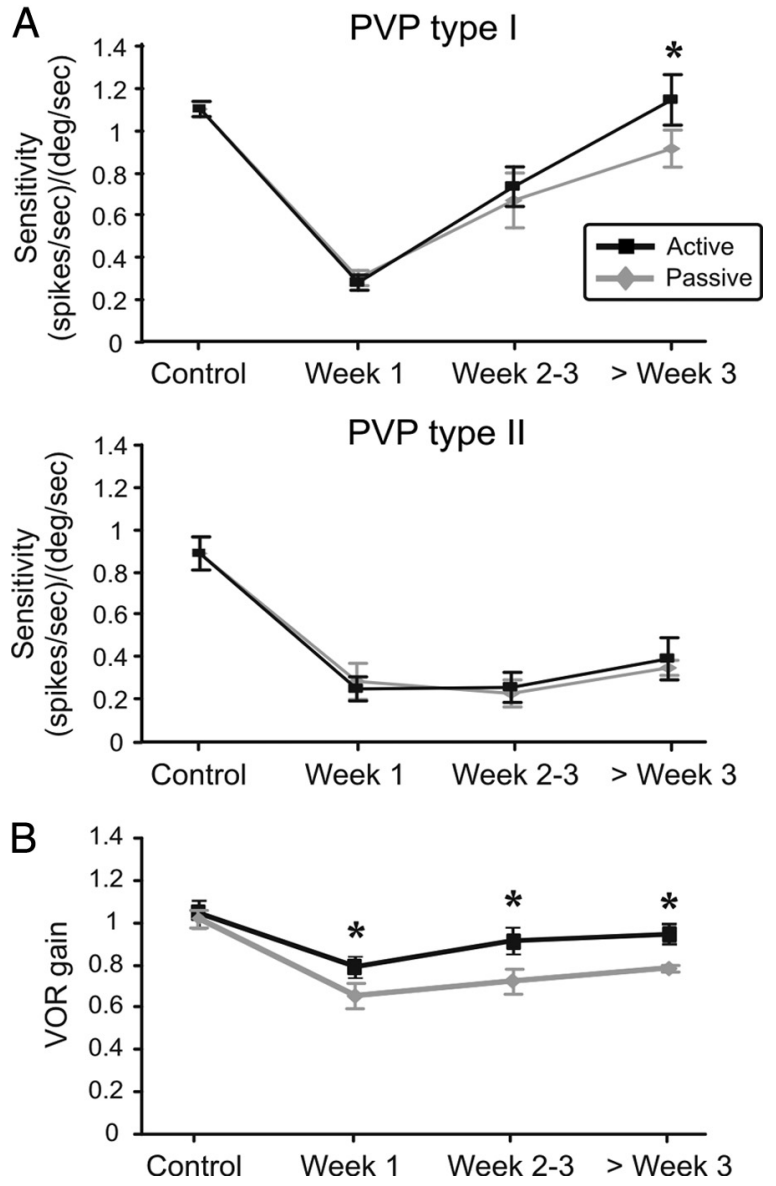

Figure 6. Comparison of average neuronal and behavioral responses during passive and active head-on-body rotations. $A$, Average sensitivities of the population of type I $(n=34)$ and type II $(n=20)$ PVP neurons during passive (gray) and active (black) movements before $(n=$ 16) and after $(n=38)$ contralateral labyrinthectomy. The difference in the sensitivity of type I PVP neurons (top panel) during active versus passive movements reached significance 3 weeks after lesion ( $20 \%$ difference; paired $t$ test, $p=0.04$ ). In contrast, there was no significant difference between the responses of type II PVP neurons during active and passive movements even 2 month after lesion (paired $t$ test, $p>0.3$ ). B, After lesion, VOR gains averaged across both animals were significantly higher during active head movements compared with similar passive rotations (paired $t$ test, $p<0.03$ ). The asterisk $\left(^{*}\right)$ indicates significant difference, paired $t$ test, $p<0.05$. Error bars indicate SEM.

evoked by passive head rotations for both animals before and after lesion (see Materials and Methods). There was no significant difference in VOR gains measured in each condition before the lesion $(p>0.9)$. However, when we compared the VOR gains measured 4 weeks after lesion, gains were significantly elevated during active (Fig. $6 \mathrm{~B}$, black) compared with passive (Fig. $6 \mathrm{~B}$, gray) rotations reaching enhancements of $>14 \%$ by week 4 (paired $t$ test, $p<0.0001$ ). Thus, our data show that an increase in VOR interneuron sensitivity is associated with improved VOR compensation and are consistent with the proposal that the measured changes in VOR responses were driven by the enhanced neuronal response sensitivities during active motion.

\section{Discussion}

To understand the neuronal basis of the impressive recovery in the VOR that occurs after vestibular loss, we examined the linkage between neuronal and behavioral responses in alert behaving monkeys. We provide the first evidence that motor learning is mediated by the dynamic reweighting of inputs from different modalities (i.e., vestibular versus extravestibular) on the single
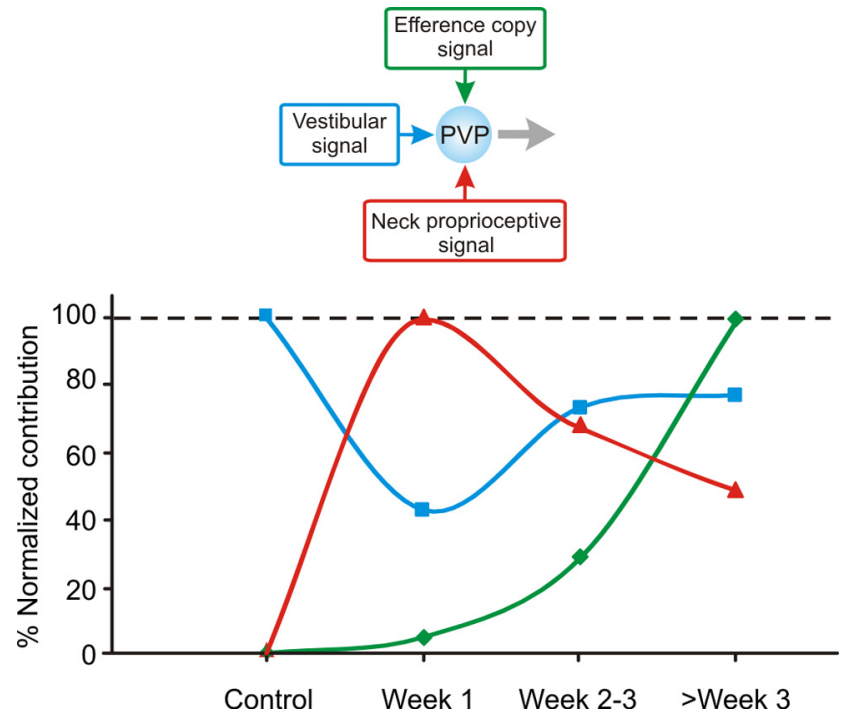

Figure 7. The time course of dynamic regulation of multimodal integration in the direct VOR pathway (i.e., type I PVP neurons) after contralateral labyrinthectomy. Responses are normalized relative to the maximum response to each of the three inputs: vestibular (blue; measured by whole-body rotation), neck proprioceptive (red; measured by body-under-head rotation), and efference copy signal (green; measured by the difference between active and passive headon-body rotation). All values are normalized relative to the maximum response (i.e., 100\%) for each input. The relative contribution of the vestibular input decreased during the first week after lesion by $>50 \%$, whereas during this same period the contribution of neck signals increased to its maximum value. Over the next weeks as the vestibular contribution returned to prelesion levels, the contribution of neck inputs decreased. Moreover, by week 3, the response of PVP neurons was enhanced during active relative to passive head-on-body movements, suggesting the additional integration of an efference copy of the neck motor command at the level of the VOR interneurons.

neurons that constitute the first stage of vestibular processing in the brain. Notably, two types of signals, not present before the lesion, were shown to have an important role in reestablishing network function. Early in the course of this process, unmasked neck proprioceptive inputs played a critical role, demonstrated by faster and more substantial recovery of vestibular sensitivities in neck proprioceptive sensitive neurons. At later stages of recovery, neurons showed enhanced responses during active head movements, as a result of the unmasking of a motor efference copy signal. Our study of the linkage between changes in neuronal response sensitivities and simultaneously measured VOR responses during passive and active head-on-body rotations with comparable trajectories demonstrated that dynamic regulation of multimodal integration (i.e., an efference copy signal) was associated with increases in the recovery of vestibular sensitivity by individual neurons. Together, our findings provide evidence, at the single-neuron level, for a functional linkage between the dynamic reweighting of extravestibular inputs and behavioral recovery, and suggest that homeostatic mechanisms underlie the unmasking of extravestibular signals at the level of VOR interneurons during motor learning.

\section{Compensatory changes in vestibular sensitivities}

The vestibular sensitivities of VOR interneurons (i.e., type I PVP neurons) showed robust recovery within 1 month (Fig. 7, blue line) consistent with the observed behavioral compensation. Previous experiments performed in anesthetized preparations (for review, see Straka et al., 2005) have reported far less neuronal recovery. However, it is important to note that synaptic inputs were likely suppressed and/or VOR interneurons could not be 
identified in these latter studies. Interestingly, we further found that neuronal recovery trailed behavioral recovery by $\sim 1$ week. One intriguing possibility is that PVP neurons consolidate adjustments previously computed elsewhere. Notably, floccular target neurons in the vestibular nuclei contribute to a parallel drive to the VOR (Broussard and Lisberger, 1992; Scudder and Fuchs, 1992). Additional studies will be required to determine whether these neurons predominantly drive the earliest phases of VOR compensation.

\section{The unmasking of extravestibular inputs}

Neurons in alert rhesus monkeys with intact vestibular function do not respond to neck proprioceptive stimulation (Roy and Cullen, 2001). After labyrinthectomy, proprioceptive responses were unmasked and were most enhanced immediately after lesion (Fig. 7, red line). Because the vestibular nuclei receive neck proprioceptive information via direct projections from the central cervical nucleus (Sato et al., 1997) and cerebellum (Eccles et al., 1974; Furuya et al., 1975; Akaike, 1983; Noda et al., 1990; Robinson et al., 1994), this suggests that the synapses mediating neck inputs are either normally silent (Kerchner and Nicoll, 2008) or, given that silent synapses are not normally abundant in the developed brain, cancelled by gating in an additional input (Keuroghlian and Knudsen, 2007). Previous studies in isolated, in vitro whole-brain preparations have characterized the synaptic efficacy of spinal inputs to the vestibular nucleus. Notably, a progressive asymmetry develops during compensation in which the synaptic efficacy decreases on the intact side and increases on the lesioned one (Vibert et al., 1999). This reorganization could potentially have more beneficial results at the cellular than network level (Rohregger and Dieringer, 2003). Our study is the first to directly measure the functional implications of the dynamic reweighting of spinal inputs to individual VOR interneurons. Although our results are also consistent with a change in the efficacy of spinal inputs, the intact side showed an increased, not decreased, sensitivity. Even more importantly, the increased response to neck proprioception, measured at the level of single neurons, was not accompanied by a parallel modality-specific improvement in motor performance. By combining neuronal and behavioral measurements, our experiments firmly establish that changes in the efficacy of spinal inputs to vestibular nucleus neurons are not linked to changes in spinal driven ocular performance (i.e., the compensatory cervico-ocular reflex).

What role does the increased efficacy of spinal inputs to vestibular nucleus neurons have in mediating vestibular compensation? As discussed above, our findings clearly show that proprioceptive inputs do not drive a compensatory eye movement to enhance motor performance. Rather, they suggest the unmasking of neck proprioceptive inputs supports a homeostatic mechanism that ensures continued dynamic stimulation of the reflex network after lesion. Evidence for a causal role of the unmasking of neck inputs in the recovery of neuronal responses was provided by two key findings. First, the resting firing rates of neck-sensitive type I PVP neurons were normal even on the first day after lesion, whereas it took $>2$ weeks for the resting discharge of neck-insensitive neurons to attain normal values. Second, we found that the recovery of neuronal sensitivity to vestibular stimulation was more rapid for our population of neck-sensitive versus neck-insensitive type I PVP neurons.

Our study is also the first to compare neuronal response sensitivities and simultaneously measured VOR responses during passive and active head-on-body rotations during vestibular compensation (Fig. 7, green line). By evaluating how neuronal responses change during actively generated versus passively applied movements, we show that the dynamic regulation of multimodal integration (in this case, an efference copy signal) can be associated with behavioral recovery. Thus, our results establish a neural correlate for the improvement in gaze stability that is observed during active motion after vestibular loss in patients and rhesus monkeys (Dichgans et al., 1973; Newlands et al., 2001; Della Santina et al., 2002). It is possible that PVP neurons might show even additional enhancement for active head motion at later stages of compensation (see trend in Fig. 7).

\section{Mechanisms for compensatory changes}

Previous studies of vestibular processing have focused on how correlation-based mechanisms (e.g., LTP and LTD) contribute to VOR plasticity. High-frequency stimulation of the vestibular nerve evokes both LTD and LTP in the vestibular nuclei (Caria et al., 1996, 2001; Grassi and Pettorossi, 2001) and the induction of either form of synaptic plasticity can be mediated through the activation of NMDA receptors (Capocchi et al., 1992; Grassi et al., 1995). The results of more recent studies have provided evidence that compensation also involves longer term changes in the vestibular nuclei including the modification of neuronal pacemaker activity (Him and Dutia, 2001) and response dynamics (Beraneck et al., 2003, 2004), as well as changes in the balance of excitatory and inhibitory inputs (Goto et al., 2000, 2001). In addition to these central compensatory mechanisms, we have described long-term changes at the level of vestibular periphery that could contribute to compensation (Sadeghi et al., 2007b).

The findings of the present study further suggest that the slower homeostatic mechanisms that promote network stability do so through the dynamic regulation of multimodal integration. One type of homeostatic plasticity that has received considerable attention is activity-dependent synaptic scaling, in which a neuron adjusts its synaptic strengths in response to changes in its own firing. Previous studies have shown that vestibular and proprioceptive inputs to the vestibular nuclei neurons are mediated by AMPA and NMDA receptors, respectively (Smith et al., 1991; Straka and Dieringer, 2004). The observation that neurons are insensitive to neck rotation before lesion suggests that these synapses are normally silent. After lesion, the increase in the number of AMPA, but not NMDA, receptors (King et al., 2002) can lead to an increase in colocalization of NMDA and AMPA receptors (Chen et al., 2000), leading to activation of "silent" NMDA synapses (Kerchner and Nicoll, 2008). In this schema, homeostatic plasticity (i.e., activation of silent synapses) and the resultant manifestation of neuronal sensitivity to neck inputs could support the long-term reweighting of synapses from vestibular inputs required for VOR compensation.

Another possible, not mutually exclusive, explanation for why extravestibular inputs are silent under normal conditions is that they are normally gated out by additional inputs. Gating mechanisms have been proposed to explain the elimination of inputs both originating from irregular afferents (Minor and Goldberg, 1991) as well as the differential processing of active and passive motion (Roy and Cullen, 2004) in vestibular pathways. Future experiments using selective manipulations of sensory inputs from each modality will be required to evaluate these potential mechanisms.

\section{Conclusion}

In closing, our findings establish at the single-unit level a functional link between the recovery of vestibular responses and the 
unmasking of extravestibular information during motor learning. Notably, the recovery of VOR motor performance involved not only the reweighting of synapses from vestibular inputs as is generally thought but also the unmasking of inputs from other modalities. Thus, this work provides a foundation for understanding the role of multimodal convergence in learning, as well as a basis for the potential development of novel rehabilitation approaches to take advantage of the convergence of sensory inputs and motor signals that contribute to the early and late stages of compensation.

\section{References}

Akaike T (1983) Electrophysiological analysis of cerebellar corticovestibular and fastigiovestibular projections to the lateral vestibular nucleus in the cat. Brain Res 272:223-235.

Beraneck M, Hachemaoui M, Idoux E, Ris L, Uno A, Godaux E, Vidal PP, Moore LE, Vibert N (2003) Long-term plasticity of ipsilesional medial vestibular nucleus neurons after unilateral labyrinthectomy. J Neurophysiol 90:184-203.

Beraneck M, Idoux E, Uno A, Vidal PP, Moore LE, Vibert N (2004) Unilateral labyrinthectomy modifies the membrane properties of contralesional vestibular neurons. J Neurophysiol 92:1668-1684.

Bronstein AM, Hood JD (1986) The cervico-ocular reflex in normal subjects and patients with absent vestibular function. Brain Res 373:399-408.

Broussard DM, Lisberger SG (1992) Vestibular inputs to brain stem neurons that participate in motor learning in the primate vestibuloocular reflex. J Neurophysiol 68:1906-1909.

Capocchi G, Della Torre G, Grassi S, Pettorossi VE, Zampolini M (1992) NMDA receptor-mediated long term modulation of electrically evoked field potentials in the rat medial vestibular nuclei. Exp Brain Res 90:546-550.

Caria MA, Melis F, Podda MV, Solinas A, Deriu F (1996) Does long-term potentiation occur in guinea-pig Deiters' nucleus? Neuroreport 7:2303-2307.

Caria MA, Melis F, Solinas A, Tavera C, Mameli O (2001) Frequencydependent LTP/LTD in guinea pig Deiters' nucleus. Neuroreport 12:2353-2358.

Chen LW, Yung KK, Chan YS (2000) Co-localization of NMDA receptors and AMPA receptors in neurons of the vestibular nuclei of rats. Brain Res 884:87-97.

Cullen KE (2008) Procedural learning. In: Cognitive psychology of memory (Roediger HL III, ed). Oxford: Elsevier.

Cullen KE, McCrea RA (1993) Firing behavior of brain stem neurons during voluntary cancellation of the horizontal vestibuloocular reflex. I. Secondary vestibular neurons. J Neurophysiol 70:828-843.

Cullen KE, Chen-Huang C, McCrea RA (1993) Firing behavior of brain stem neurons during voluntary cancellation of the horizontal vestibuloocular reflex. II. Eye movement related neurons. J Neurophysiol 70:844-856.

Cullen KE, Rey CG, Guitton D, Galiana HL (1996) The use of system identification techniques in the analysis of oculomotor burst neuron spike train dynamics. J Comput Neurosci 3:347-368.

Della Santina CC, Cremer PD, Carey JP, Minor LB (2002) Comparison of head thrust test with head autorotation test reveals that the vestibuloocular reflex is enhanced during voluntary head movements. Arch Otolaryngol Head Neck Surg 128:1044-1054.

Dichgans J, Bizzi E, Morasso P, Tagliasco V (1973) Mechanisms underlying recovery of eye-head coordination following bilateral labyrinthectomy in monkeys. Exp Brain Res 18:548-562.

Duensing F, Schaefer KP (1958) The activity of single neurons in the region of vestibular nuclei in horizontal acceleration, with special reference to vestibular nystagmus (in German). Arch Psychiatr Nervenkr Z Gesamte Neurol Psychiatr 198:225-252.

Eccles JC, Sabah NH, Táboríková H (1974) The pathways responsible for excitation and inhibition of fastigial neurones. Exp Brain Res 19:78-99.

Feldman DE (2009) Synaptic mechanisms for plasticity in neocortex. Annu Rev Neurosci 32:33-55.

Furuya N, Kawano K, Shimazu H (1975) Functional organization of vestibulofastigial projection in the horizontal semicircular canal system in the cat. Exp Brain Res 24:75-87.
Goto F, Straka H, Dieringer N (2000) Expansion of afferent vestibular signals after the section of one of the vestibular nerve branches. J Neurophysiol 84:581-584.

Goto F, Straka H, Dieringer N (2001) Postlesional vestibular reorganization in frogs: evidence for a basic reaction pattern after nerve injury. J Neurophysiol 85:2643-2646.

Grassi S, Pettorossi VE (2001) Synaptic plasticity in the medial vestibular nuclei: role of glutamate receptors and retrograde messengers in rat brainstem slices. Prog Neurobiol 64:527-553.

Grassi S, Della Torre G, Capocchi G, Zampolini M, Pettorossi VE (1995) The role of GABA in NMDA-dependent long term depression (LTD) of rat medial vestibular nuclei. Brain Res 699:183-191.

Grassi S, Frondaroli A, Pessia M, Pettorossi VE (2001) Exogenous glutamate induces short and long-term potentiation in the rat medial vestibular nuclei. Neuroreport 12:2329-2334.

Hayes A, Richmond B, Optican L (1982) A UNIX-based multiple process system for real-time data acquisition and control. WESCON Conf Proc 2:1-10.

Him A, Dutia MB (2001) Intrinsic excitability changes in vestibular nucleus neurons after unilateral deafferentation. Brain Res 908:58-66.

Huterer M, Cullen KE (2002) Vestibuloocular reflex dynamics during highfrequency and high-acceleration rotations of the head on body in rhesus monkey. J Neurophysiol 88:13-28.

Jürgens R, Mergner T (1989) Interaction between cervico-ocular and vestibulo-ocular reflexes in normal adults. Exp Brain Res 77:381-390.

Kerchner GA, Nicoll RA (2008) Silent synapses and the emergence of a postsynaptic mechanism for LTP. Nat Rev Neurosci 9:813-825.

Keuroghlian AS, Knudsen EI (2007) Adaptive auditory plasticity in developing and adult animals. Prog Neurobiol 82:109-121.

King J, Zheng Y, Liu P, Darlington CL, Smith PF (2002) NMDA and AMPA receptor subunit protein expression in the rat vestibular nucleus following unilateral labyrinthectomy. Neuroreport 13:1541-1545.

Kotak VC, Fujisawa S, Lee FA, Karthikeyan O, Aoki C, Sanes DH (2005) Hearing loss raises excitability in the auditory cortex. J Neurosci 25:3908-3918.

Lorente de Nó R (1933) Vestibular-ocular reflex arc. Arch Neurol Psychiatry 30:245-291.

Maffei A, Turrigiano GG (2008) Multiple modes of network homeostasis in visual cortical layer 2/3. J Neurosci 28:4377-4384.

Malinvaud D, Vassias I, Reichenberger I, Rössert C, Straka H (2010) Functional organization of vestibular commissural connections in frog. J Neurosci 30:3310-3325.

McCrea RA, Strassman A, Highstein SM (1987) Anatomical and physiological characteristics of vestibular neurons mediating the vertical vestibuloocular reflexes of the squirrel monkey. J Comp Neurol 264:571-594.

Minor LB, Goldberg JM (1991) Vestibular-nerve inputs to the vestibuloocular reflex: a functional-ablation study in the squirrel monkey. J Neurosci 11:1636-1648.

Newlands SD, Hesse SV, Haque A, Angelaki DE (2001) Head unrestrained horizontal gaze shifts after unilateral labyrinthectomy in the rhesus monkey. Exp Brain Res 140:25-33.

Noda H, Sugita S, Ikeda Y (1990) Afferent and efferent connections of the oculomotor region of the fastigial nucleus in the macaque monkey. J Comp Neurol 302:330-348.

Robinson FR, Phillips JO, Fuchs AF (1994) Coordination of gaze shifts in primates: brainstem inputs to neck and extraocular motoneuron pools J Comp Neurol 346:43-62.

Rohregger M, Dieringer N (2003) Postlesional vestibular reorganization improves the gain but impairs the spatial tuning of the maculo-ocular reflex in frogs. J Neurophysiol 90:3736-3749.

Roy JE, Cullen KE (1998) A neural correlate for vestibulo-ocular reflex suppression during voluntary eye-head gaze shifts. Nat Neurosci 1:404-410.

Roy JE, Cullen KE (2001) Selective processing of vestibular reafference during self-generated head motion. J Neurosci 21:2131-2142.

Roy JE, Cullen KE (2002) Vestibuloocular reflex signal modulation during voluntary and passive head movements. J Neurophysiol 87:2337-2357.

Roy JE, Cullen KE (2004) Dissociating self-generated from passively applied head motion: neural mechanisms in the vestibular nuclei. J Neurosci 24:2102-2111.

Sadeghi SG, Minor LB, Cullen KE (2006) Dynamics of the horizontal vestibuloocular reflex after unilateral labyrinthectomy: response to high fre- 
quency, high acceleration, and high velocity rotations. Exp Brain Res 175:471-484

Sadeghi SG, Chacron MJ, Taylor MC, Cullen KE (2007a) Neural variability, detection thresholds, and information transmission in the vestibular system. J Neurosci 27:771-781.

Sadeghi SG, Minor LB, Cullen KE (2007b) Response of vestibular-nerve afferents to active and passive rotations under normal conditions and after unilateral labyrinthectomy. J Neurophysiol 97:1503-1514.

Sadeghi SG, Mitchell DE, Cullen KE (2009) Different neural strategies for multimodal integration: comparison of two macaque monkey species. Exp Brain Res 195:45-57.

Sato H, Ohkawa T, Uchino Y, Wilson VJ (1997) Excitatory connections between neurons of the central cervical nucleus and vestibular neurons in the cat. Exp Brain Res 115:381-386.

Scudder CA, Fuchs AF (1992) Physiological and behavioral identification of vestibular nucleus neurons mediating the horizontal vestibuloocular reflex in trained rhesus monkeys. J Neurophysiol 68:244-264.

Shelhamer M, Robinson DA, Tan HS (1992) Context-specific adaptation of the gain of the vestibulo-ocular reflex in humans. J Vestib Res 2:89-96.

Shimazu H, Precht W (1966) Inhibition of central vestibular neurons from the contralateral labyrinth and its mediating pathway. J Neurophysiol 29:467-492.

Smith PF, de Waele C, Vidal PP, Darlington CL (1991) Excitatory amino acid receptors in normal and abnormal vestibular function. Mol Neurobiol 5:369-387.

Straka H, Dieringer N (2004) Basic organization principles of the VOR: lessons from frogs. Prog Neurobiol 73:259-309.

Straka H, Vibert N, Vidal PP, Moore LE, Dutia MB (2005) Intrinsic membrane properties of vertebrate vestibular neurons: function, development and plasticity. Prog Neurobiol 76:349-392.

Sylvestre PA, Cullen KE (1999) Quantitative analysis of abducens neuron discharge dynamics during saccadic and slow eye movements. J Neurophysiol 82:2612-2632.

Sylvestre PA, Cullen KE (2006) Premotor correlates of integrated feedback control for eye-head gaze shifts. J Neurosci 26:4922-4929.

Vibert N, Babalian A, Serafin M, Gasc JP, Mühlethaler M, Vidal PP (1999) Plastic changes underlying vestibular compensation in the guinea-pig persist in isolated, in vitro whole brain preparations. Neuroscience 93: 413-432. 